\title{
A Monolithic MQW InP/InGaAsP-Based Comb Generator
}

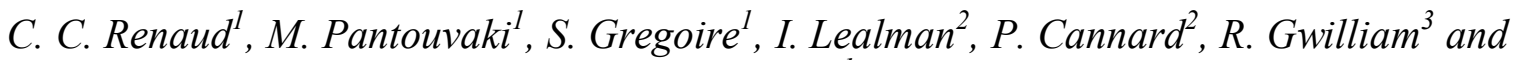 \\ A. J. Seeds ${ }^{1}$ \\ ${ }^{1}$ Department of Electronic and Electrical Engineering, University College London, Torrington \\ Place, London, WC1E 7JE, United Kingdom
}

${ }^{2}$ Centre for Integrated Photonics, Ipswich, IP5 3RE, United Kingdom

${ }^{3}$ Ion Beam Centre, University of Surrey, Guildford Surrey, GU2 7RX, United Kingdom

\begin{abstract}
We report a monolithic optical frequency comb generator using quaternary/quaternary multiple quantum well InP/InGaAsP material as phase modulator and gain medium in a Frequency Modulated (FM) laser design. The modulation was generated by quantum confined Stark effect to achieve a comb-line spacing of $\mathbf{2 4 . 4}$ GHz. The laser was fabricated using a single epitaxial growth step and quantum well intermixing to realize low loss phase and modulation sections. The resulting comb generator produces lines with a spacing exactly given by the modulation frequency, differential phase noise between adjacent lines of $-82 \mathrm{dBc} / \mathrm{Hz}$ at $1 \mathrm{kHz}$ offset and a comb spectrum width of up to $2 \mathrm{THz}$.
\end{abstract}

Index Terms - Optical frequency comb generation, Laser diode, FM laser.

\section{INTRODUCTION}

Many different applications, ranging from Dense WDM optical communications, to photonic $\mathrm{THz}$ synthesis require a reliable and cost effective frequency reference source. Atomic or molecular resonances are commonly used to provide absolute frequency references, however they are limited to a few particular frequencies [1]. To overcome such limitation several other solutions have been proposed to provide a regular spaced frequency comb, such as a Fabry-Perot interferometer or a fibre ring resonator [2]. Unfortunately the frequency accuracy and stability of these devices are limited by the optical length of the resonators. Another solution is the use of deep angle modulation of an optical source to generate precisely spaced frequency lines [3]. However the power and number of frequency lines and their spacing are limited by the difficulty of realising such modulation at high frequency. Other solutions such as an amplified fibre loop comb generator [4] or mode locked semiconductor lasers [5] can offer hundreds of lines with a spacing from 10 to $25 \mathrm{GHz}$ over a band of at least $1 \mathrm{THz}$ with a stability limited by the thermal stability of the semiconductor laser (master laser in the case of the fibre loop).
The principle of the FM laser [6] is an extension of the mode-locked laser solution and should offer a large number of equally spaced frequency lines, spaced by the FM source frequency, over the gain spectrum of the semiconductor laser, but without the strong intensity modulated envelope which is detrimental to some filtering schemes. In this paper we describe such a laser using Quantum Confined Stark Effect (QCSE) [7] as the refractive index modulation mechanism in a monolithic InP/InGaAsP based laser. The laser described in this paper provides lines spaced by $24.4 \mathrm{GHz}$ over a $15 \mathrm{~nm}$ wavelength $(2 \mathrm{THz})$ span. It showed good stability performance and an almost constant output power. Furthermore, the phase noise generated when heterodyning two successive lines was limited by the phase noise of the electrical reference source used.

\section{PRINCIPLE OF THE FM SOURCE}

As stated above FM operation laser occurs when a laser cavity phase is modulated at a frequency close to the axial frequency [6]. At this point of operation, one will observe a continuous wave $(\mathrm{CW})$ output as there is no amplitude modulation and the spectrum will comprise a number of lines depending on the frequency detuning from the axial mode and the amplitude of the modulation. When the modulation frequency is equal to the axial frequency the laser will transfer to a modelocked pulse regime [6]. The FM laser regime is generated by the phase modulation which induces coupling between the different modes of the cavity. The resulting spectrum can be described by the following equations:

$$
\begin{aligned}
& E(t)=E_{0} \sum_{n=-\infty}^{\infty} J_{n}(\Gamma) e^{j\left(\omega_{0}+n \omega_{m}\right) t} \\
& \Gamma=\frac{\Delta \Phi}{2 \pi} \frac{\omega_{a x}}{\left|\omega_{a x}-\omega_{m}\right|}
\end{aligned}
$$


In these equations $\mathrm{E}_{0}$ is the amplitude of the optical field, $\omega_{0}$ is the laser operating frequency, $\omega_{\mathrm{m}}$ is the modulation frequency, $\omega_{\mathrm{ax}}$ is the axial mode spacing of the Fabry-Perot cavity, $\Delta \Phi$ is the amplitude of the phase modulation and $J_{n}$ is an ordinary Bessel function. Note that these equations represent an ideal laser with a flat gain spectrum and pure single mode operation. A long cavity semiconductor laser will typically be multimode and absorb more light at the short wavelength end of the spectrum, thus affecting the resulting spectrum. The perfect theoretical laser will generate a spectrum as shown in Fig 1.

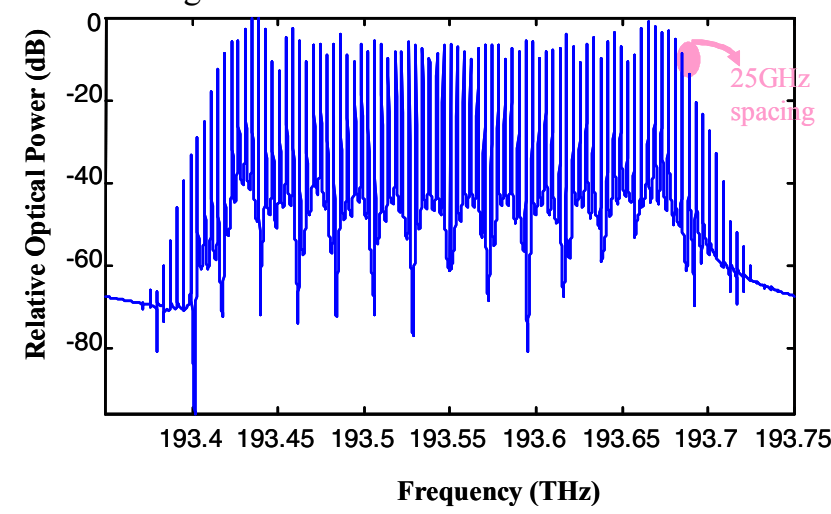

Fig. 1. Theoretical spectrum of an FM laser.

\section{DESCRIPTION OF THE LASER}

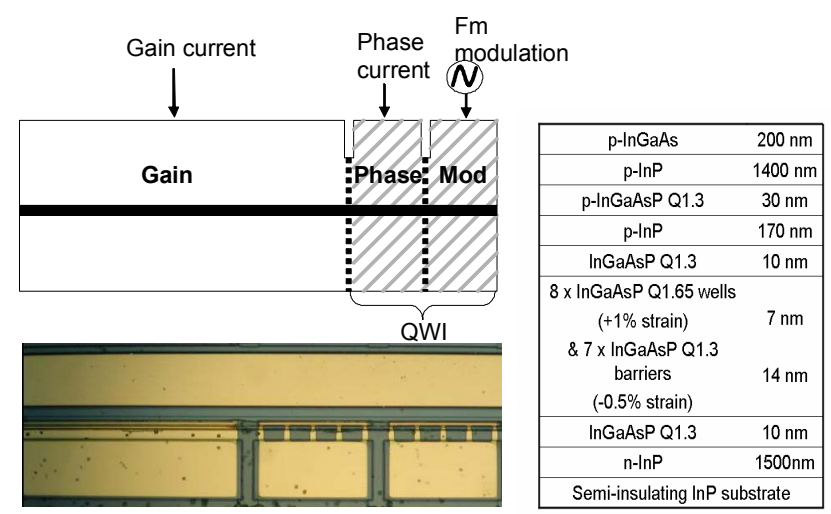

Fig. 2. Schematic description of the three section laser (left) and epitaxial structure (right).

Fig 2 (left) shows a schematic of the three section laser. The laser was $1.85 \mathrm{~mm}$ long ( $25 \mathrm{GHz}$ axial mode spacing) with $70 \mu \mathrm{m}$ long modulation and phase sections. The phase section was use to change the axial mode spacing thus the frequency detuning while the modulation frequency could remain constant. Fig. 2 (right) shows the epitaxial structure of the material used for the laser. The structure was grown by metal-organic vapour phase epitaxy (MOVPE) on a semi-insulating InP substrate. The active region consists of eight $7 \mathrm{~nm}$-wide compressively strained (1\%) InGaAsP Q1.65 wells, alternating with seven $14 \mathrm{~nm}$-wide tensile strained (0.5\%) InGaAsP Q1.3 barriers, sandwiched between two $10 \mathrm{~nm}$-thick InGaAsP Q1.3 waveguide layers. On top of the InGaAs contact layer, a $0.5 \mathrm{~m}$ InP buffer layer and a final $0.1 \mathrm{~m} \mathrm{InGaAs} \mathrm{buffer} \mathrm{layer} \mathrm{were} \mathrm{grown.} \mathrm{They}$ were used to protect the contact cap from damage as shallow ion implantation was used to generate QWI as in [8]. For this work we implanted $8 \times 10^{14} \mathrm{P}$ ions at $100 \mathrm{keV}$ energy and $200{ }^{\circ} \mathrm{C}$. With this implantation energy damage was restricted to the buffer layer, thus when it was subsequently removed the surface quality of the contact cap was intact. The wafer was masked with 600 $\mathrm{nm}$ of PECVD SiO2 during implantation in order to protect the gain sections. After implantation the wafer was rapidly thermally processed at $650{ }^{\circ} \mathrm{C}$ for 90 seconds which created a $35 \mathrm{~nm}$ blue-shift of the bandgap in the phase and modulator sections. Part of the wafer was then processed into PIN devices, and photocurrent spectra were measured to extract the spectral dependence of the material absorption as a function of reverse bias. Such a measurement allowed the estimation of the effect of QCSE on refractive index. This showed that the refractive index for the propagating mode will change by 0.03 with $2 \mathrm{~V}$ reverse Bias. Such an index change is sufficient to create the desired FM laser effect.

The fabricated laser was a ridge waveguide design with oxide-bridged contacts in order to reduce the capacitance of the modulator section (picture in Fig 2.). The different sections were separated by an isolation trench through the highly doped top layers. The oxide-bridged contact for the modulation section allowed a $380 \mathrm{fF}$ measured capacitance and a $20 \Omega$ series resistance resulting in a maximum modulation bandwidth of $42 \mathrm{GHz}$. As both phase section and modulation section were intermixed, the laser threshold remained relatively low at $80 \mathrm{~mA}$ for a $1.88 \mathrm{~mm}$ long device. The laser was operated at 200 $\mathrm{mA}$ bias current giving a total output of $1 \mathrm{~mW}(0.5 \mathrm{~mW}$ coupled into a single mode fibre).

\section{EXPERIMENTS AND RESULTS}

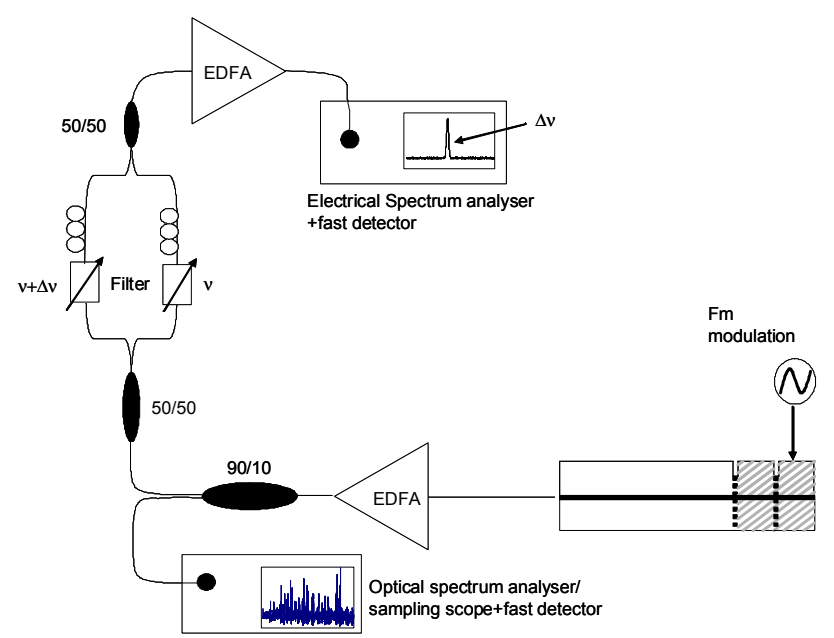

Fig. 3. Systems used for experimental measurements

Fig 3 shows the different systems used to characterize the FM laser. First the output spectrum of the laser was assessed with a spectrum analyzer. Finer spectral studies were also done with a Fabry-Perot interferometer with a spectral resolution of $10 \mathrm{MHz}$. The laser output power 
stability was also studied using a fast photodetector (50 $\mathrm{GHz}$ bandwidth) and a sampling oscilloscope. This allowed intensity modulation effects on the laser output to be studied. The stability of the line spacing and its relation to the frequency source were assessed by heterodyning two adjacent lines and sending the resulting signal to the fast photodetector which was connected to a spectrum analyser. Note that in this case Filters were used at the output of the erbium doped fibre amplifiers (EDFA) to reduce the amplified spontaneous emission (ASE) level in the resulting spectrum.
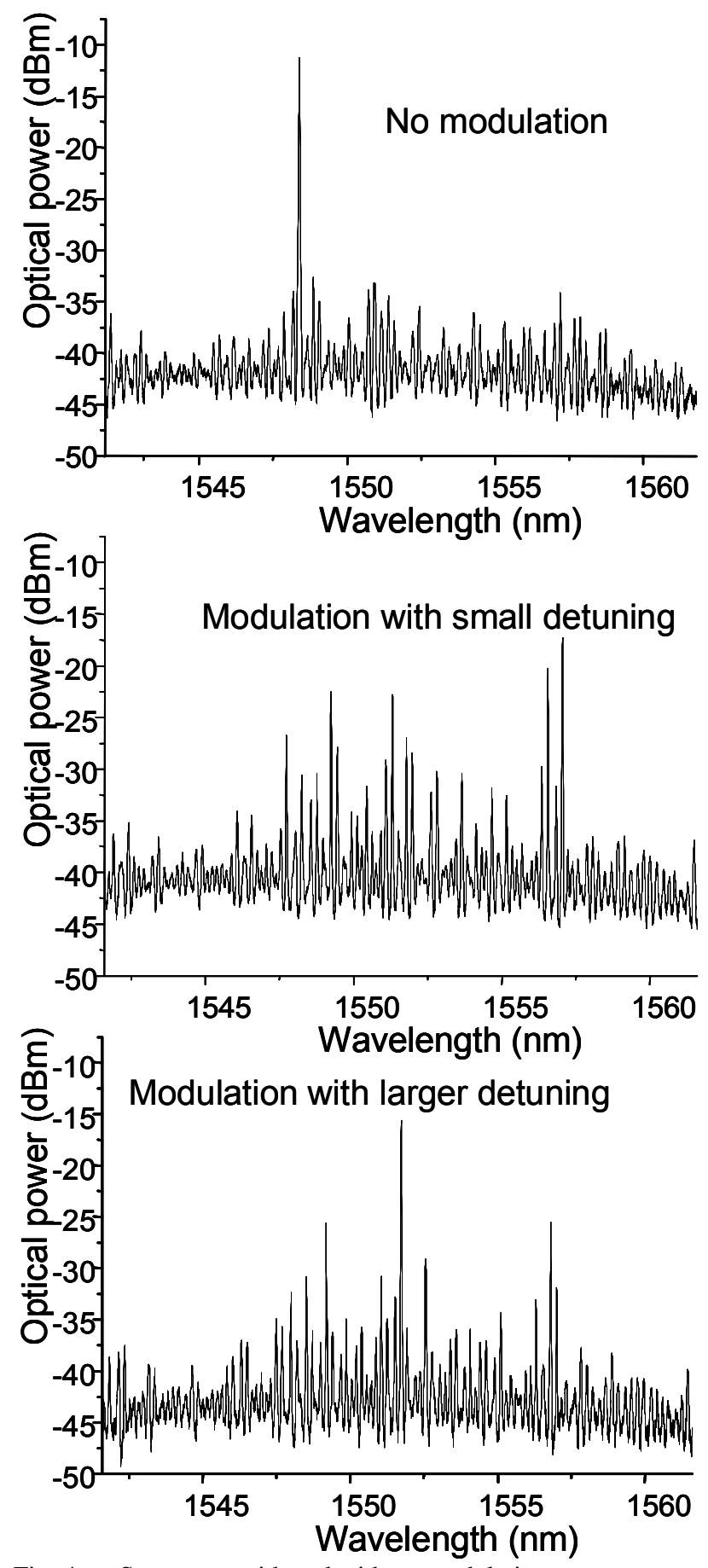

Fig. 4. Spectrums with and without modulation
Fig. 4 shows the output spectrum of the laser with and without $24.4 \mathrm{GHz}$ modulation $(2 \mathrm{~V}$ peak to peak with $2 \mathrm{~V}$ negative bias). With no modulation lasing was on a single longitudinal mode. With modulation the spectrum shows a large number of lines appearing across the spectrum. When the phase was changed in order to detune the laser axial frequency further from the modulation frequency the number of lines diminished and were closer to the original peak frequency as expected for a FM laser. Note that the spectrum is not symmetric as for the ideal example given previously. This is mainly due to the strong absorption on the short wavelength side of the spectrum. Furthermore, the trenches used to isolate the different sections were inducing small reflection thus creating a system of coupled Fabry-Perot cavities. This induced higher losses in the cavity at frequency spacings determined by the length of the modulation section. This was reflected in the optical spectrum by the lack of lines created in some part of it.

To assess the quality of the created comb lines their linewidth was measured with a Fabry-Perot interferometer with a $10 \mathrm{MHz}$ resolution. The nonmodulated laser had a measured linewidth of $14 \mathrm{MHz}$. When modulated the laser was creating lines with the same measured linewidth.

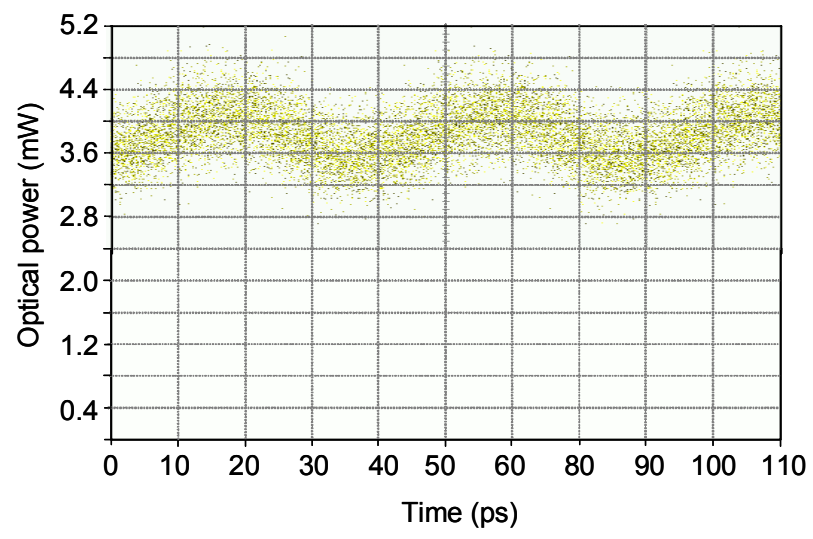

Fig. 5. Output power as a function of time

To confirm that the device was operating as an FM laser the stability of its output power was measured with a fast photodetector both on a spectrum analyzer and on a sampling oscilloscope. Fig. 5 shows the result obtained with the sampling oscilloscope. As expected there is a slight amplitude modulation as the QCSE slightly changes the absorption of the modulation section. The spectrum analyzer did show a weak peak at $24.4 \mathrm{GHz}$ in agreement with the previous measurement.

In order to fully assess the FM operation, the comb line spacing should be measured and its relation to the modulation frequency should be demonstrated. In order to do this final measurement we used the heterodyne system described previously with $0.5 \mathrm{~nm}$ wide line filters on each branch of the system. 


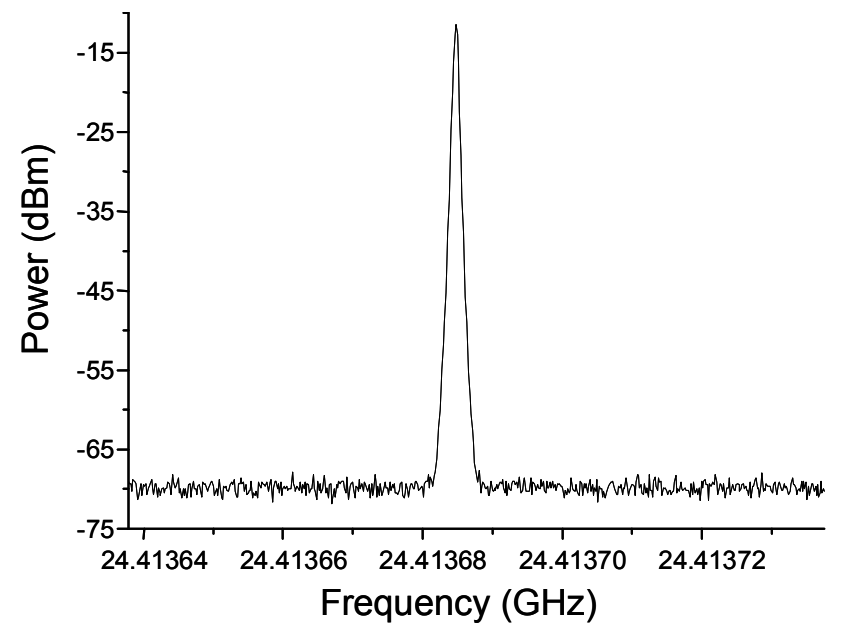

Fig. 6. electrical spectrum of the heterodyne signal created from two adjacent lines of the comb generator.

The result is shown in Figure 6 for one given pair of lines. For this measurement the spectrum analyser span was $100 \mathrm{kHz}$ and the resolution bandwidth was $1 \mathrm{kHz}$. The line was at the exact frequency of the modulation source and had the same spectral purity as expected. Thus as the comb lines were spaced by the modulation frequency, we could definitely conclude the device was operating as a FM laser. Phase noise measurements were also made on the heterodyne signal to compare it with the frequency source. It showed that no added phase noise was coming from the laser and that the stability of the line spacing was given by the frequency source. Typically the phase noise was $-82 \mathrm{dBc} / \mathrm{Hz}$ at $1 \mathrm{kHz}$ and $108 \mathrm{dBc} / \mathrm{Hz}$ at $1 \mathrm{MHz}$.

\section{CONCLUSION}

We have demonstrated FM operation in a monolithic semiconductor laser. This optical frequency combgenerator offers $24.4 \mathrm{GHz}$ spaced lines over a spectral width of $15 \mathrm{~nm}(\sim 2 \mathrm{THz})$. The laser lines were exactly spaced by the modulation frequency and the residual intensity modulation was less than $20 \%$. Each line had the same linewidth of $14 \mathrm{MHz}$ as the non modulated laser. When heterodyned two adjacent line gave a high purity frequency with phase noise limited by the frequency source. This achievement was possible using a combination of different fabrication techniques on the quaternary/quaternary $\mathrm{InGaAsP} / \mathrm{InP}$ material. First the bandgap of part of the wafer was blue shifted using QWI by shallow ion implant and RTP. The $35 \mathrm{~nm}$ bandgap shift obtained allowed low signal absorption in the phase and modulation sections of the device. Secondly the fabrication of the device was made using oxide-bridge techniques to reduce the capacitance of the modulation section to $380 \mathrm{fF}$. This allowed for an efficient reverse bias modulation using QCSE at $24.4 \mathrm{GHz}$ which was within the bandwidth of the modulation section (42 $\mathrm{GHz}$ ). Such monolithic comb generators provide a compact alternative to fibre loop techniques with larger comb line output powers than passive phase modulator techniques.

\section{REFERENCES}

[1] A. J. Lucero Y. C. Chung, R. W. Tkach,"Survey of atomic transitions for absolute frequency locking of lasers for lightwave systems," IEEE Photonics Technol. Letters, vol. 3, pp.484-486, 1991

[2] N. Shimosaka, K. Kaede, M. Fujiwara, S. Yamazaki, S. Murata, M. Nishio, "Frequency separation locking and synchronization for FDM optical sources using widely frequency tunable laser diodes," Sel. Area Com., vol. 8, pp. 1078-1086, 1990

[3] M. Kourogi, K. Nakagawa, M. Ohtsu, "Wide-span optical frequency comb generator for accurate optical frequency difference measurement," IEEE journal of Quantum Electron., vol. 29, pp. 26932701, 1993

[4] S. Bennet, B. Cai, E. Burr, O. Gough, A. J. Seeds, "Terahertz, zero frequency error, tunable optical comb generator for DWDM applications," Paper WM5 OFC'99, San-Diego, 1999

[5] M. Teshima, M. Koga, K. Sato, “Accurate frequency control of a mode-locked laser diode by referencelight injection," Optics Letters, vol. 22, pp. 126-128, 1997

[6] D. J. Kuizenga, A. Siegman, "FM-laser operation of the Nd:YAG laser," IEEE journal of Quantum Electron., vol. 6, pp. 673-677, 1970.

[7] B. Cai, A. J. Seeds, J. S. Roberts, "MQW tuned semiconductor lasers with uniform frequency response," IEEE Photonics Technol. Letters, vol. 6, pp. 496-498, 1994

[8] E. J. Skogen, J. W. Raring, J. S. Barton, L. A. Johansson, L. A. Coldren, "Postgrowth Control of the Quantum-Well Band Edge for the Monolithic Integration of Widely Tunable Lasers and Electroabsorption Modulators," IEEE J. of Selected Topic in Quantum Electron., vol. 9, pp. 1183-1190, 2003 